\title{
The reproducibility of heart Deformation Analysis for the evaluation of global cardiac function
}

\author{
Eileen Hu-Wang ${ }^{1 *}$, Haimanot Wasse ${ }^{2}$, Jeremy Collins ${ }^{3}$, James C Carr ${ }^{3}$, Craig B Langman ${ }^{1}$, Michael Markl ${ }^{3}$, Kai Lin ${ }^{3}$ \\ From 19th Annual SCMR Scientific Sessions \\ Los Angeles, CA, USA. 27-30 January 2016
}

\section{Background}

Analysis of global cardiac function is important for cardiovascular risk estimation but can be time-consuming. Using deformable image registration (DIR) algorithms, Heart Deformation Analysis (HDA) is a recently developed method for measuring cardiac function and motion on existing cardiac cine MR images. With a totally automatic workflow, HDA has the potential to be useful in clinical practice. In this study, the inter-study reproducibility of Heart Deformation Analysis will be analyzed.

\section{Methods}

With IRB approval, 17 healthy volunteers were recruited to participate in the study. Each subject underwent two cardiac MR scans, the second scan taking place 14 days after the first scan. All participants underwent MRI examinations using the same cardiac cine protocol on a $1.5 \mathrm{~T}$ scanner (Avanto, Siemens AG, Germany). At fourchamber, two-chamber, and short-axis views, Segmented bSSFP cine sequences were acquired in the cardiac imaging planes with breath-holding. Imaging parameters were as follows: $\mathrm{TR} / \mathrm{TE}=2.8 / 1.1 \mathrm{~ms}$; flip angle $=65^{\circ}$, voxel size $=2.1 \times 2.1 \times 8.0 \mathrm{~mm}^{3}$, bandwidth $=930 \mathrm{~Hz} /$ pixel. Eight to ten short-axis myocardial slices were set to cover the entire LV from base to apex. Each cine acquisition was acquired with retrospective ECG-gating and reconstructed into 25 phases. Left ventricular ejection fraction (LVEF) and left ventricular mass (LV mass) were automatically calculated by using the HDA tool. LVEF and LV mass were correlated between the two scans using SPSS software. SPSS intraclass correlation coefficient (ICC) was used to assess variations in LVEF and LV mass values between scans, with an alpha of 0.05 chosen to demarcate statistical significance.

${ }^{1}$ Northwestern University Feinberg School of Medicine, Chicago, IL, USA Full list of author information is available at the end of the article

\section{Results}

A moderate to high correlation was found between LVEF and LV mass between the first and second scans. The LVEF ICC was found to be $0.636, p=0.03$. The LV mass ICC was found to be $0.527, p=0.08$. See figure 1

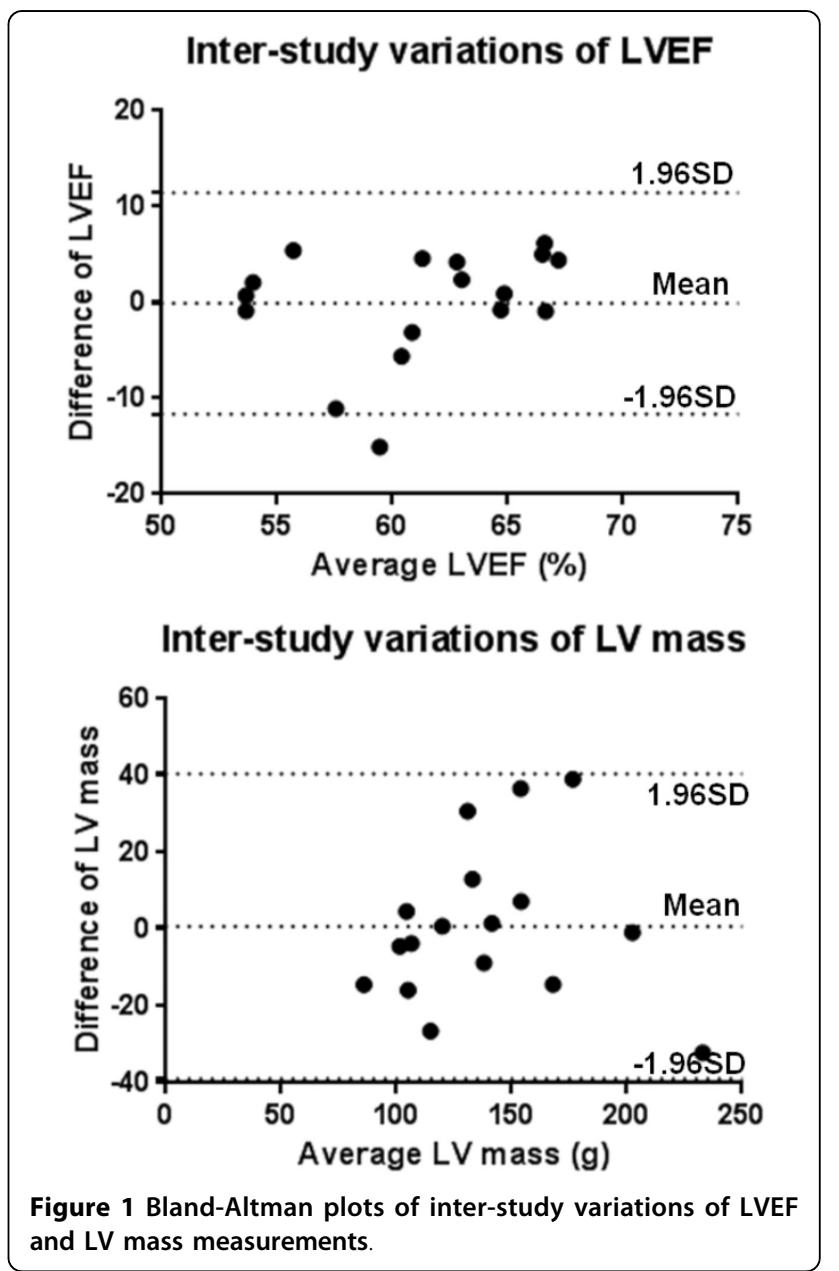


for the Bland-Altman plots showing inter-study variations of LVEF and LV mass measurements.

\section{Conclusions}

Good inter-study reproducibility was found for evaluating global cardiac function. A moderate to high correlation was found between LVEF $($ ICC $=0.636)$ and LV mass $(\mathrm{ICC}=0.527)$ between the first and second scans. There was statistical significance for correlation of LVEF between scans $(p=0.03)$ but no statistical significance was found for correlation of LV mass between the two scans $(\mathrm{p}=0.08)$. The LV mass correlation was probably not found to be significant in this study because of the small sample size of 17 subjects; it is possible that a larger sample size could increase statistical power of the study.

In conclusion, the inter-study reproducibility and totally automatic workflow of HDA give this analysis method the potential to be useful in clinical practice, where a more time-efficient analysis method can be more easily incorporated into patient care.

\section{Authors' details}

${ }^{1}$ Northwestern University Feinberg School of Medicine, Chicago, IL, USA. ${ }^{2}$ Department of Nephrology, Northwestern University Feinberg School of Medicine, Chicago, IL, USA. 'Department of Radiology, Northwestern University Feinberg School of Medicine, Chicago, IL, USA.

Published: 27 January 2016

\section{Submit your next manuscript to BioMed Central} and take full advantage of:

- Convenient online submission

- Thorough peer review

- No space constraints or color figure charges

- Immediate publication on acceptance

- Inclusion in PubMed, CAS, Scopus and Google Scholar

- Research which is freely available for redistribution

Submit your manuscript at www.biomedcentral.com/submit 